\title{
Morphological characterization and impedance spectroscopy study of porous 3D carbons based on graphene foam-PVA/phenol-formaldehyde resin composite as electrode material for supercapacitors
}

\author{
Bello Abdulhakeem*, Barzegar Farshad, Momodu Damilola, Taghizadeh Fatimeh, Fabiane Mopeli, Dangbegnon \\ Julien, Manyala Ncholu*
}

\begin{abstract}
The design and fabrication of porous electrode materials is highly desirable for improving the performance of electrochemical supercapacitors (ECs) and thus, it is important to produce such porous materials in large quantities. In this study, we used a microwave method to produce porous carbonaceous materials designated as graphene foam/polyvinyl alcohol/formaldehyde (GF/ PVA/F) and graphene foam- polyvinyl alcohol/phenol-formaldehyde (GF/PVA/PF) from graphene foam, phenol formaldehyde and polyvinyl alcohol (PVA). Scanning electron microscopy (SEM), Raman spectroscopy and Fourier-Transform Infrared Spectroscopy (FTIR) were used to characterize the surface morphology, structural defects and functional groups of the materials respectively. Based on these porous materials, the two symmetrical ECs fabricated exhibited a specific capacitance in the range of 0.62-1.92 $\mathrm{F}$ $\mathrm{cm}^{-2}$, phase angles of $-81^{\circ}$ and $-84^{\circ}$ and resistor-capacitor (RC) relaxation time constants of 4 and 14 seconds. The physicochemical properties of the electrolyte ion (diffusion) and its influence on the capacitive behavior of the porous materials were elucidated. These encouraging results demonstrate the versatile potential of these porous materials (GF/PVA/F and GF/PVA/PF) in developing high energy storage devices.
\end{abstract}

Keywords: Graphene foam, KOH activation, porous morphology, porous electrode, supercapacitors

\section{Introduction}

Electrochemical energy storage systems such as electrical double layer capacitors (EDLCs) often called supercapacitors or ultracapacitors, are considered to be leading candidates in high power devices. ${ }^{1}$ They store charge by creating a double layer of opposite charge separation at the interface between the electrolyte and the conductive electrode material, offering a remarkably high power density. Thus they are not affected by charge transfer kinetics and hence can be rapidly charged and discharged, which gives the advantage of a long cycle life compared to batteries. ${ }^{1}$ However, unlike their counterparts, namely batteries, supercapacitors store less charge per unit volume and hence research on supercapacitors is geared towards improving in electrochemical energy storage materials with a focus on the storage capacity or voltage since the energy is a function of the voltage squared. ${ }^{2}$

Conventional EDLCs are mostly based on carbon electrodes such as activated carbon, ${ }^{3}$ carbide-derived carbon (CDC), ${ }^{4,5}$ onion-like carbon, ${ }^{6}$ carbon nanotubes $(\mathrm{CNT}),{ }^{7,8}$ and more recently graphene, ${ }^{9,10}$ which usually have a low frequency response due to ohmic resistance contribution from all the components of the cell, such as the electrolyte, electrode thickness and separator. For AC-line filtering, capacitors need to respond harmonically at $120 \mathrm{~Hz}$. However, at this frequency EDLCs are not capable of this; rather they behave like a transmission line, acting like resistors instead of capacitors. ${ }^{11}$ This is usually ascribed to unsuitable pore structure and distribution, leading to a high rate of diffusion and charge transfer.
The recent boom in graphene research has aroused much interest in graphene-based multifunctional composite/hybrid or aerogel materials which combine the functions of several components as electrode materials. ${ }^{12}$ Different compositions of multifunctional materials are possible, but the materials that are most suitable used materials as electrodes are composites of materials with relevant properties such as electrical conductivity, porosity and surface area for improved energy performance. Pore size distribution, surface area and the size of the electrolyte ions could influence the electrochemical performance of the composite significantly. For this reason the optimization of micro/nano-porous architecture and the design of materials for improving performance is the subject of ongoing research.

Double layer porous structures of carbons or carbon hydrogels are attractive choices for electrode materials with practical importance because of their high porosity, large surface area and electrical conductivity. ${ }^{13,14}$ These structures are produced by different techniques which may include hydrothermal polymerization and carbonization of mixtures of polymers and carbon from different sources ${ }^{15}$ and template-directed growth. ${ }^{16}$ Recently, a threedimensional graphene/polymer-based hydrogel with exceptional properties was reported by Zhang et. al. ${ }^{17}$ The porous materials produced demonstrated a superior supercapacitor performance in ionic liquid with a specific capacitance of $92 \mathrm{~F} / \mathrm{cm}^{3}$ and $39 \mathrm{Wh} / \mathrm{liter}$ in $\mathrm{EMIMBF}_{4}$ electrolyte respectively. Wei et $a .^{15}$ also reported hydrochars that were efficiently transformed into microporous 
carbons via hydrothermal carbonization. The material produced exhibited a capacitance value up to $175 \mathrm{~F} / \mathrm{g}$ at an ultra-high current density of $20 \mathrm{~A} / \mathrm{g} .{ }^{15}$ Yamada et al ${ }^{18}$ reported porous carbon materials for EDLCs exhibiting a specific capacitance of $120 \mathrm{~F} / \mathrm{g}$ in

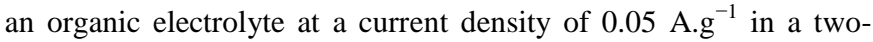
electrode configuration. The high capacitance performance was attributed to the interconnected hierarchical porous structures of the materials. Porous carbon electrodes in an aqueous electrolyte (such as $\mathrm{KOH}$, a pore-expanding agent) with different molar concentrations, coupled with exceptional performance, including high capacitance value and energy density, were also reported. ${ }^{9,19}$ The performance was ascribed to the hierarchical pore structure and interconnected mesopores with high surface area Furthermore, as stated earlier, significant efforts have been devoted to producing fast EDLCs for AC-line filtering. Miller et al ${ }^{21}$ reported EDLCs electrodes made from vertically oriented nanosheets on heated nickel substrate by radio frequency (RF) plasma enhanced chemical vapor deposition with an exceptional time constant of 200 microseconds, and more recently Ren et $a{ }^{20}$ reported perpendicularly-oriented graphene grown on nickel foam with a cell areal capacitance of 0.32 $\mathrm{mF} \mathrm{cm}^{-2}$. In spite of these efforts in the development of these electrodes, rather slow progress has been demonstrated towards the commercialization of EDLCs.

In this paper, we report on a two-step green chemistry synthesis of highly porous carbon structures, using a microwave irradiation process to produce a hydrogel based on graphene foam by the addition of polyvinyl alcohol (PVA), polypyrrole (PPY), phenol and formaldehyde. These precursors were selected because they are environmentally friendly and low-cost carbon sources that can be used to produce microporous and mesoporous with quite large specific surface area, high electrical conductivity and subsequently used as polarizable electrodes in electrochemical supercapacitors yielding high capacitance, high working voltage with low leakage current. $^{21-23}$ The carbonization/activation of the hydrogel with $\mathrm{KOH}$ is aimed at producing highly porous carbon electrode materials to address some of the challenges encountered with EDLCs. Our choice of phenol and formaldehyde as additional carbon precursors is due to the fact they have been shown to produce mesoporous materials. ${ }^{23,24}$ The electrochemical characteristics of symmetrical supercapacitor cells produced from these materials in aqueous $\mathrm{KOH}$ is presented. This work is aimed mostly at the studying the frequency response of the material to series capacitance, equivalent series resistance (ESR) and the phase angle for potential application as an electrode for high performance supercapacitors.

\section{Experimental}

\section{Synthesis of graphene foam (GF)}

Graphene foam (GF) was prepared by chemical vapor deposition (CVD) onto a catalytic nickel foam (Alantum Innovations in Alloy Foam Munich, Germany) as reported in our previous work. ${ }^{25}$ Typically, graphene was grown on nickel foam template, after which the nickel was removed. To provide mechanical support for the graphene during the removal of the nickel, polymethylmethacrylate (PMMA) was drop coated on the sample and baked at $180{ }^{\circ} \mathrm{C}$ for 30 minutes. Thereafter the samples were placed in a $3 \mathrm{M} \mathrm{HCl}$ solution at $80{ }^{\circ} \mathrm{C}$ overnight to ensure complete removal of the nickel template. The resulting GF samples were placed in acetone at $50{ }^{\circ} \mathrm{C}$ for 30 minutes to remove the PMMA. They were then rinsed in deionised water and dried.

\section{Synthesis and activation of composites hydrogel}

Three-dimensional hydrogel was synthesized via microwave treatment as follows: $5 \mathrm{mg}$ of GF produced by CVD above was dispersed in a solution containing $0.1 \mathrm{~g} / \mathrm{ml}$ of PVA in a glass vial by ultrasonication to obtain a good homogeneous dispersion of graphene sheets and $1.5 \mathrm{ml}$ of $\mathrm{HCl}$ was added as a cross linker. ${ }^{17}$ After obtaining the dispersed solution, $0.5 \mathrm{ml}$ of formaldehyde was added to obtain a mixture of GF/PVA/F. To obtain the second sample of GF/PVA/FP, $0.1 \mathrm{~g}$ of phenol was added to the previous solution of GF/PVA/F. Both solutions were treated by microwave to obtain the hydrogel. Prior to microwave treatment, $0.5 \mathrm{ml}$ of a conducting polymer polypyrole (PPY) was poured into both solutions in order to enhance the conductivity of the final product. PPY was chosen because of its high density and conductivity when compared to other conducting polymers. ${ }^{26}$ In addition, PPY undergoes a fast redox reaction which improves the electrochemical performance of the composite material. ${ }^{27}$ After the addition of PPY, the solution was stirred for 30 minutes to obtain a homogeneous dispersion and the resulting solution was transferred into a microwave reactor chamber operated at $180{ }^{\circ} \mathrm{C}$ for 6 hours. The resulting hydrogel was washed with deionized water and dried for 6 hours.

The as-prepared hydrogel was then soaked in aqueous $\mathrm{KOH}$ solution (with a $\mathrm{KOH} /$ hydrogel mass ratio of 7 ) for 24 hours, to form the hydrogel $\mathrm{KOH}$ mixture for chemical activation. ${ }^{10}$ The composite mixture was then placed in a horizontal tube furnace ramped from room temperature to $800{ }^{\circ} \mathrm{C}$ at $10{ }^{\circ} \mathrm{C} /$ minute under argon flow and annealed for 1 hour for the activation process. This process transforms the composite hydrogel into carbon materials consisting of a continuous pore network. The activated material denoted as GF/PVA/F and GF/PVA/PF was washed with deionised water and dried at $120^{\circ} \mathrm{C}$ for 12 hours after which samples were characterized.

\section{Materials characterization}

The surface area measurements of the produced samples were performed by liquid nitrogen cyrosorption at $77 \mathrm{~K}$ using Micromeritics TriStar II 3020 (version 2.00). The morphologies of the activated porous carbons were observed by scanning electron microscopy (SEM: Zeiss Ultra Plus 55 field emission scanning electron microscope (FE-SEM) operated at an accelerating voltage of $2.0 \mathrm{kV}$ ). Raman spectroscopy measurements were carried out using a Jobin Yvon Horiba TX 6400 micro-Raman spectrometer equipped with a triple monochromator system to eliminate contributions from Rayleigh lines and using LabSpec (Ver. 5.78.24) analytical software. All the samples were analyzed with a $514 \mathrm{~nm}$ argon excitation laser $(1.5 \mathrm{~mW}$ laser power on the sample to avoid thermal effects). Fourier-Transform Infrared (FTIR) spectra were recorded from a Vertex 70v (Bruker) spectrometer in the 4000-600 
$\mathrm{cm}^{-1}$ range with $4 \mathrm{~cm}^{-1}$ resolution and analyzed with the Opus software

\section{Coin cell fabrication and electrochemical characterization}

For electrode fabrication best practice methods were used to obtain reliable results and draw conclusions. Some of these recommendations for industrial applications involve using a mass of active electrode between 10 and $20 \mathrm{mg}$, an electrode thickness between 100 and $200 \mu \mathrm{m}$ and a two electrode configuration cell measurement. $^{28,29}$ This configuration gives the real physical and practical configuration, internal voltages and the charge transfer that takes place in a packaged cell. It thus provides the best indication of an electrode material's performance rather than the three-electrode configuration which is valuable for determining specific material characteristics. ${ }^{29}$ Our experimental data are therefore based on realtime devices using industrial best practice methods.

The electrode materials which consisted of the activated material and Polyvinylidene difluoride (PVDF) binder with a weight ratio of 9: 1 were homogenized in an agate mortar. The mixture was then dissolved in 1-methyl-2-pyrrolidinone (NMP) to form a paste which was pressed on nickel foam, which acts as a current collector. A uniform film coating with a thickness of $0.2 \mathrm{~mm}$ was coated on the current collector and dried at $60^{\circ} \mathrm{C}$ in an oven for 8 hours to ensure complete evaporation of the NMP. The weight of activated material in each electrode with a diameter of $16 \mathrm{~mm}$ is at least $\sim 9 \mathrm{mg}$. Coin cells were made using a glass microfiber filter paper as the separator in $6 \mathrm{M} \mathrm{KOH}$ aqueous electrolyte. Finally, electrochemical performance tests of a symmetrical two-electrode configuration were carried out using a Bio-logic SP-300 potentiostat. Electrochemical measurement includes cyclic voltammetry (CV), galvanostatic charge-discharge (GCD) and electrochemical impedance spectroscopy (EIS). The CV tests were carried out in the potential range of 0 to $1 \mathrm{~V}$ at different scan rates ranging from $10 \mathrm{~m} \mathrm{Vs}^{-1}$ to $100 \mathrm{~m} \mathrm{Vs}^{-1}$. The GCD measurements were also carried out at different current densities from $3.7 \mathrm{~mA} \mathrm{~cm} \mathrm{~cm}^{-2}\left(0.5 \mathrm{~A} \mathrm{~g}^{-1}\right)$ to $7.5 \mathrm{~mA}$ $\mathrm{cm}^{-2}\left(1 \mathrm{~A} \mathrm{~g} \mathrm{~g}^{-1}\right)$ and the EIS studies were carried out in the frequency range of $100 \mathrm{kHz}-10 \mathrm{mHz}$ with a $2 \mathrm{mV}$ ac amplitude.

\section{Results and discussion}

\section{Structure and morphology of the activated material}

The nitrogen adsorption-desorption isotherms were used to obtain information about the pore size distribution and Brunauer-EmmettTeller (BET) specific surface area of the samples and shown in Figure 1. As observed in Figure 1a, the BET specific surface area of sample GF/PVA/FP is higher than that of GF/PVA/F with both samples exhibiting a type II/IV isotherm and corresponding specific surface area values of $705 \mathrm{~m}^{2} \mathrm{~g}^{-1}$ with $80 \%$ micropores distribution and $610 \mathrm{~m}^{2} \mathrm{~g}^{-1}$ with a micropores distribution of $58 \%$, respectively. It is suggested that the addition of phenol to the sample leads to higher micropores distribution and surface area. The pore size distribution of both samples is shown in Figure $1 \mathrm{~b}$ with an average pore size distribution centred at $\sim 4 \mathrm{~nm}$. The increase in the pore distribution below $2 \mathrm{~nm}$ is evidence indicating the existence of micropores in this sample. $^{30}$
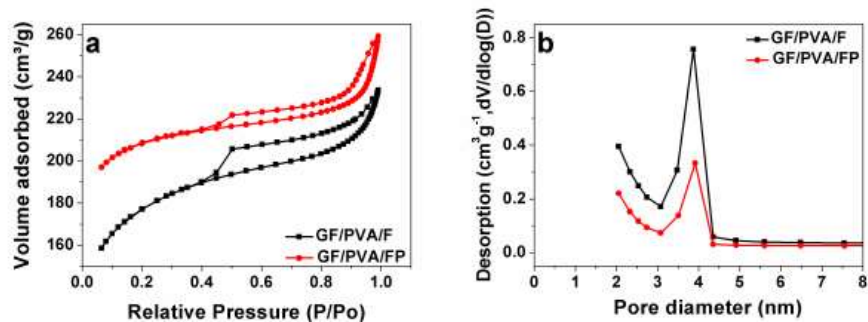

Figure 1 (a) Nitrogen adsorption-desorption isotherm loops and (b) the BJH pore size distribution curves, calculated from desorption branch of isotherm.

SEM micrographs of samples GF/PVA/F and GF/PVA/FP are shown in Figure 2. Figure 2 (a) and (b) are the low- and highmagnification images of GF/PVA/F respectively. They exhibit porous sponge-like cavities at the surface, with the average diameters of the cavities in the order of hundreds of nanometers. In addition, the high magnification image shows that these cavities are superficial on the whole material. Figure 2 (c) and (d) present the low- and high-magnification images of GF/PVA/FP respectively. They clearly show more uniformly distributed porous cavities in the sponge-like morphology and corroborate the results observed from the BET measurements. The distribution of uniform pores is higher in the GF/PVA/FP and could facilitate a rapid mass diffusion during operation, suitable for practical application as electrode materials. ${ }^{30}$
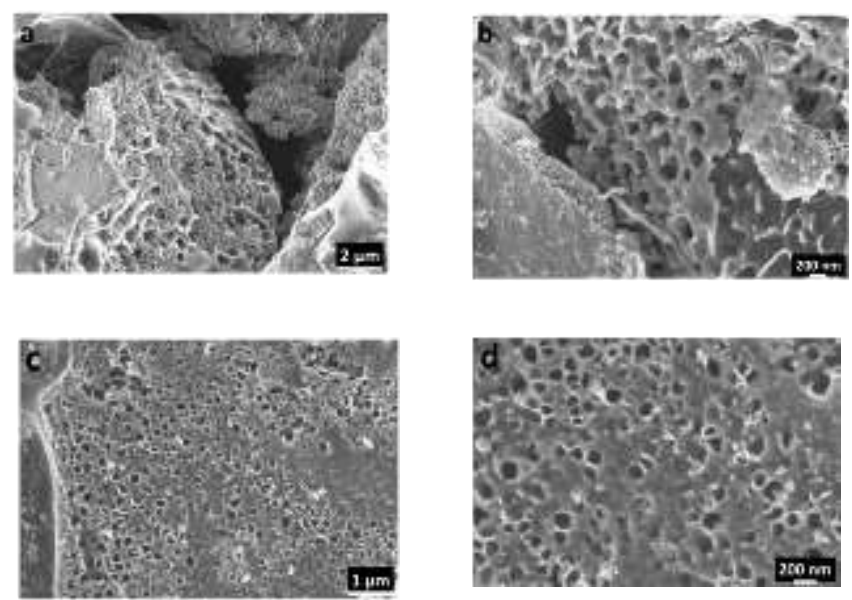

Figure 2 SEM images (a) low magnification and (b) high magnification of GF/PVA/F and (c) low magnification and (d) high magnification of GF/PVA/FP

Raman spectroscopy has been shown to be an important technique for the characterization of structural defects in carbon materials. ${ }^{31}$ Figure 3 (a) presents typical Raman spectra of the graphene foam (GF) and the as-prepared porous materials. The Raman spectrum of the GF, with prominent peaks at $1582.2 \mathrm{~cm}^{-1}$ and $2705.7 \mathrm{~cm}^{-1}$, corresponding to the $\mathrm{G}$ and 2D-bands of graphitic material with the absence of the D-peak (disorder) at $1350 \mathrm{~cm}^{-1}$, showed that the GF 
produced was of good quality. The spectra of the activated materials (GF/PVA/F and GF/PVA/FP) show significantly pronounced features at $\sim 1345 \mathrm{~cm}^{-1}, \sim 1582 \mathrm{~cm}^{-1}$ and $\sim 2692 \mathrm{~cm}^{-1}$ which also correspond to the $\mathrm{D}, \mathrm{G}$ and $2 \mathrm{D}$-bands of graphitic material. The appearance of the D-peak in the samples could be attributed to activation process or to interactions between the PVA, GF, F and P during microwave treatment, which could also introduces a strain effect and results in the shift of the 2D-peak position as observed. ${ }^{32}$ The G-band originates from the in-plane vibration of $\mathrm{sp}^{2}$ carbon atoms and is a doubly degenerate (TO and LO) phonon mode $\left(\mathrm{E}_{2 \mathrm{~g}}\right.$ symmetry) at the Brillouin zone center. ${ }^{31}$ The D-peak, which is attributed to the breathing modes of $\mathrm{sp}^{2}$ rings is activated by a double resonance effect in the presence of defects. ${ }^{33}$ The 2D-peak is the second order of the D-peak. The relatively high intensity of the Dband in the Raman spectra is attributed to the presence of a large amount of disorder within the bulk of the activated material. From these spectra we conclude that the structural unit of our activated material mostly consists of defected/deformed carbon.
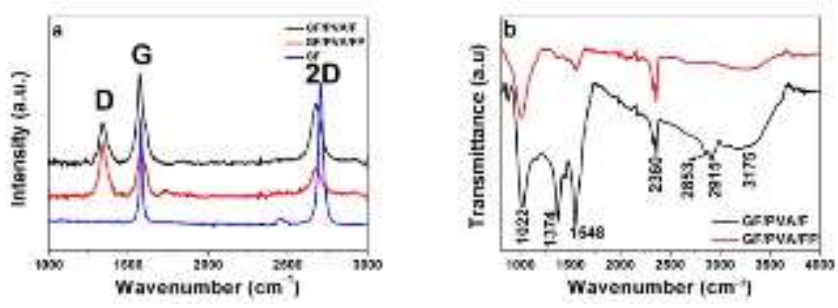

Figure 3 (a) Raman and (b) FTIR spectra of GF/PVA/F and GF/PVA/FP

FTIR was performed to identify the presence of the functional groups in both samples and the results are presented in Figure 3 (b). Both spectra exhibit quite similar peaks with the GF/PVA/F showing additional peaks at 2853 and $2915 \mathrm{~cm}^{-1}$ these are attributed to the asymmetrical and symmetrical stretching of $-\mathrm{CH}_{2}$ respectively. In the GF/PVA/FP spectrum these two peaks are absent, which may be due to the reaction of aldehydes (formaldehyde) with alcohols (phenol) in an acidic environment, producing other functional groups consisting of $-\mathrm{OH}$ and -OR bonded to the carbon. In general, the solubility of phenols in water is poor, hence the addition of $\mathrm{HCl}$ to improve its solubility which also renders the solution acidic. ${ }^{34}$ The general reaction between aldehydes and alcohols can be written as follows:

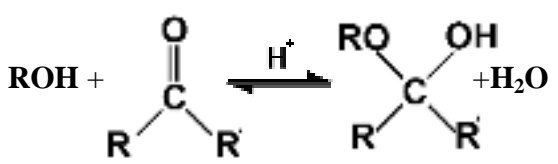

The peak at $1022 \mathrm{~cm}^{-1}$ is due to the infrared vibration of the $\mathrm{C}-\mathrm{O}$ bond. Moreover, peaks between $1374-1548 \mathrm{~cm}^{-1}$ were observed which are probably due to the aromatic $\mathrm{C}=\mathrm{C}$ bond. In addition, the peaks at $2360 \mathrm{~cm}^{-1}$ were attributed to $\mathrm{C}-\mathrm{H}$ vibrational modes and the broad peak at $3175 \mathrm{~cm}^{-1}$ is attributed to the symmetrical stretching vibration of $-\mathrm{OH}$ groups ${ }^{35}$.

\section{Electrochemical characterization}

Symmetrical cells of GF/PVA/F and GF/PVA/FP were assembled without the addition of a conductive agent such as carbon black or acetylene black due to the conductivity of our materials. The CV measurements of these materials in $6 \mathrm{M}$ $\mathrm{KOH}$ electrolyte are shown in Figure 4. Figure 4 (a) presents the $\mathrm{CV}$ curves of activated material based on GF/PVA/F, while Figure 4 (b) presents the $\mathrm{CV}$ curves based on GF/PVA/FP at scan rates from 20 to $100 \mathrm{mV} \mathrm{s}^{-1}$. Both samples exhibit similar rectangular shapes over the range of scan rates $\left(20\right.$ to $100 \mathrm{mV} \mathrm{s}^{-}$ ${ }^{1}$ ), with the cells still maintaining their quasi-rectangular shapes as the scan rate increases. This indicates excellent rate charge propagation and rate performance at the electrode/electrolyte interface, ${ }^{36}$ thus confirming the formation of an efficient EDLCs. ${ }^{6}$ Areal capacitances of 0.25 and $0.43 \mathrm{~F} \mathrm{~cm}^{-2}$ were obtained for GF/PVA/F and GF/PVA/FP respectively at a scan rate of $20 \mathrm{mV} \mathrm{s}^{-1}$. The higher capacitance value of the GF/PVA/FP cell is attributed to a more uniform distribution of porous cavities which function as channels and facilitate ion transport or adsorption at the electrode/electrolyte interface, leading to improved capacitance performance. It also worth stating that when the uniform porous electrode is saturated with the electrolyte, the ions are already located on the electrode/electrolyte interface and are easily pushed off and attracted from the electrode on a small displacement, giving high mobility which might advance fast and efficient charge propagation, leading to an increase in the capacitance. ${ }^{30}$
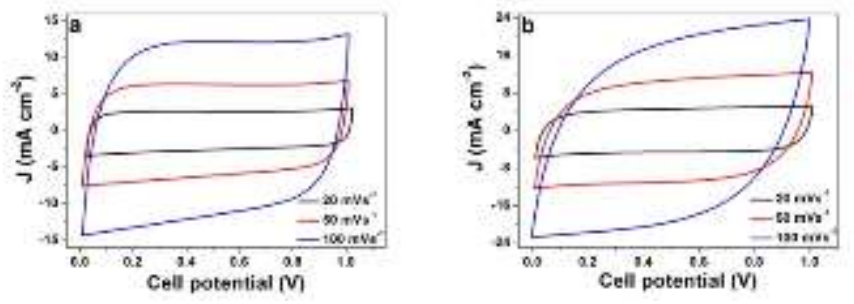

Figure 4 (a) CV curves of GF/PVA/F cell at scan rates 20, 50, and $100 \mathrm{~m} \mathrm{Vs}^{-1}$ (b) CV curves of GF/PVA/FP cell at scan rates 20, 50, and $100 \mathrm{~m} \mathrm{Vs}^{-1}$.

Chronopotentiometry studies were carried out to evaluate the specific capacitance and cycling stability of the cells. Figures 5 (a) and (b) present the charge-discharge (CD) curves of the cells of both materials containing $\sim 18 \mathrm{mg} \mathrm{cm}^{-2}$ of the activated materials at current densities of 3.7 and $7.5 \mathrm{~mA} \mathrm{~cm}^{-2}$ respectively between 0 and $1 \mathrm{~V}$. The symmetrical characteristics of the CD curves of both cells measured at different current densities are linear, demonstrating an ideal double-layer behavior with the absence of any faradaic processes. This indicates that porous electrodes based on these activated materials have excellent rate performance and a rapid ion transport mechanism. ${ }^{36}$ The cell capacitance is calculated from the discharge curve using equation 1 :

$C=\frac{I}{d V / d t}$ 
Where $I$ is the discharge current in amperes (A), and $d V / d t$ is the slope in volt per second $\left(\mathrm{Vs}^{-1}\right)$ after the Ohmic drop to $0.5 \mathrm{~V} \cdot{ }^{37} \mathrm{In}$ a two electrode symmetrical cell the specific capacitance of a single electrode in $\mathrm{F} \mathrm{cm}^{-2}$ of the active material from the $\mathrm{CD}$ is related to the capacitance of the cell equation 2 :

$$
C_{s p}=\frac{2 J t}{V}
$$

where $J$ is the response current density $\left(\mathrm{mA} \mathrm{cm}^{-2}\right), V$ is the potential window in volts (V) and $t$ is the discharge time in seconds (s). From this formula the specific capacitance of a single electrode for each of the cells was calculated to be $0.28 \mathrm{~F} \mathrm{~cm}^{-2}$ (gravimetric specific capacitance: $158 \mathrm{~F} \mathrm{~g} \mathrm{~g}^{-1}$ ) and $0.47 \mathrm{~F} \mathrm{~cm}^{-2}$ (gravimetric specific capacitance: $177 \mathrm{~F} \mathrm{~g}^{-1}$ ) respectively at a current density of $3.7 \mathrm{~mA}$ $\mathrm{cm}^{-2}$. These values are similar to the results obtained from the $\mathrm{CV}$ measurements and higher than the values reported for mesoporous carbon materials as electrodes for electrochemical supercapacitors. ${ }^{38}$ These results can be compared with the values obtained by Wang et. $a l{ }^{39}$ from chrysanthemum like carbon nanofiber foam architecture supercapacitors on highly porous nickel foams synthesized under ambient pressure chemical vapor deposition (APCVD) with $1.37 \mathrm{~F}$ $\mathrm{cm}^{-2}$ (specific capacitance: $23.83 \mathrm{~F} \mathrm{~g}^{-1}$ ) at a current density of 1.78 $\mathrm{mA} \mathrm{cm}^{-2}$. They can also be compared with the perpendicularly oriented graphene sheets reported by Ren et. al. ${ }^{20}$ which exhibit a capacitance of $0.83 \mathrm{mF} \mathrm{cm}$ at a scan rate of $1 \mathrm{Vs}^{-1}$ for a single electrode as well as with the carbon nanofiber supercapacitors with porous nickel foam obtained via CVD reported by McDonough et. $a l .{ }^{40}$ with areal capacitance of $1.2 \mathrm{~F} \mathrm{~cm}^{-2}$.
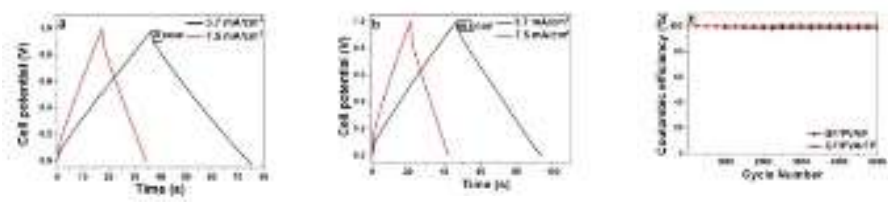

Figure $5(\mathrm{a}, \mathrm{b}) \mathrm{CD}$ curves of GF/PVA/F and GF/PVA/FP cells at current densities of $3.7 \mathrm{~mA} \mathrm{~cm}{ }^{-2}$ and $7.5 \mathrm{~mA} \mathrm{~cm}^{-2}$. (c) Coulombic efficiency at a current density of $3.7 \mathrm{~mA} \mathrm{~cm}^{-2}$

Long term cycling performance is another parameter for estimating the stability of EDLCs. Continuous CD experiments were therefore carried out over 5000 cycles at a current density of $3.7 \mathrm{~mA} \mathrm{~cm}^{-2}$ as shown in Figure 5 (c). The figure shows that the coulombic efficiency of the GF/PVA/F cell is $98 \%$ which means that the cell loses about $2 \%$ of its initial capacitance after 5000 cycles and the Coulombic efficiency of the GF/PVA/FP cell is $100 \%$ which means that there was no capacitance loss from the first cycle. However, after about 3000 cycles the Coulombic efficiency of the GF/PVA/FP cell gradually increases by $\sim 1 \%$. Although this increase is very small, it may be due to the high uniform pore distribution which functions as a series of channels and may promote ion intercalation into these pores, creating more nano-pores after many $\mathrm{CD}$ cycle leading to more accessible surface area and hence an increase in the efficiency. $^{20}$
The dependence of the equivalent resistance or voltage drop (IR) of the cell was also calculated from Figure 5 (a) and (b) using equation $3:^{37}$

$$
E S R=\Delta U_{D r o p} / 2 I
$$

where $\Delta U_{\text {Drop }}$ is the voltage drop at the beginning of the discharge curve in volts $(\mathrm{V})$ measured at a constant current $I$ in amperes (A). The equivalent series resistance (ESR) values estimated from the IR drop using equation 4 are $5.7 \Omega$ for GF/PVA/F and $4.0 \Omega$ for GF/PVA/FP.

\section{Electrochemical impedance spectroscopy (EIS) analysis}

EIS is an important parameter for investigating the electrical conductivity of electrodes. We measured the impedance of the electrode materials in the frequency range of 0.01 to $10^{5} \mathrm{~Hz}$ with 2 $\mathrm{mV}$ AC signal amplitude. The complex plane (Nyquist) plots are presented in Figure 6 for both electrode materials. Both plots show a semicircle in the high frequency region and a nearly vertical line in the low-frequency region as observed in the inset to Figure 6 (a).
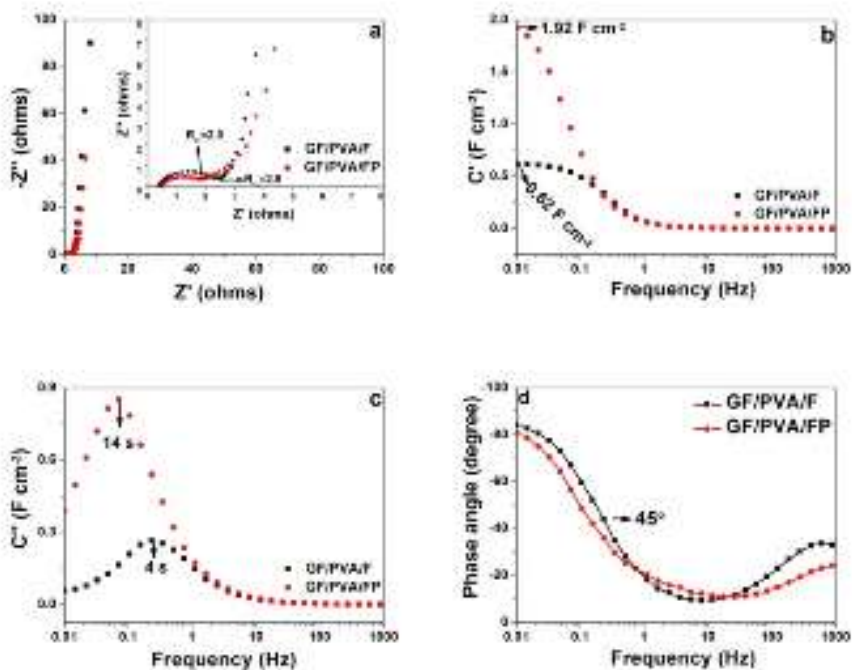

Figure 6 Comparison of EIS characteristics of the cells: (a) Nyquist plot with inset showing the semicircle, (b) the real part of the cells capacitance against frequency, (c) the imaginary part of the cells capacitance against frequency and (d) the phase angle versus frequency for both GF/PVA/F and GF/PVA/FP.

The high frequency region is related to the electronic resistance within the electrode materials. The vertical line in the low frequency region is a result of the frequency dependence of ion diffusion at the electrolyte/electrode interface. This vertical line, which is parallel to the imaginary axis, reflects the high conductivity and rate capabilities of the electrode materials and also indicates ideal capacitor behavior. In the high frequency region, the values obtained from the $\mathrm{x}$-axis intercept of the Nyquist plot correspond to the ESR of the electrode material and the electrolyte, ${ }^{41}$ while the high to mid frequency region gives the charge transfer resistance $R_{c t}$. Figure 6 (a) 
compares the Nyquist plots of both cells. The intercepts on the $\mathrm{x}$-axis of the Nyquist plots give the ESR for both cells which are $0.38 \Omega$ for GF/PVA/F and $0.45 \Omega$ for GF/PVA/FP. These values indicate excellent ion diffusion of the electrode materials. Also, from the inset to Figure 6 (a) it is observed that the addition of phenol decreases the Warburg region and the value of the charge transfer resistance $\mathrm{R}_{\mathrm{ct}}$ from the loop in the mid to high frequency region from $2.5 \Omega$ to $\sim 2.0 \Omega$. The ESR estimated from the IR drop (Figure 5) are generally higher when compared with values obtained from the intercepts on the $\mathrm{x}$-axis of the Nyquist plots. This might be due to the fact that IR drop values calculated from CD represents the sum of the resistances of the various components in the cell, meaning the addition of resistance of the active material and the separator and coin steel, whereas values obtained from the Nyquist plots are a function of frequency which is a more sensitive to the active material and thus more accurate parameter for estimating the ESR.

The frequency response of porous carbon electrodes has been modelled with a single series resistor-capacitor (RC) circuit. ${ }^{6,37,42}$ Based on this model, the resistance in the real part of the impedance (Nyquist) plot, the capacitance can be calculated using equation $4 .{ }^{37}$

$$
C=-1 /\left(\omega Z^{\prime \prime}\right)
$$

The values of the real and imaginary parts of the capacitance, $C^{\prime}(\omega)$ and $C^{\prime \prime}(\omega)$ are calculated according to equation 5 (a-c). $C^{\prime}(\omega)$ represents the real accessible capacitance of the cell at the corresponding frequency which represent the deliverable capacitance, while $C^{\prime \prime}(\omega)$ represents the energy loss due to the irreversible process of the electrodes. ${ }^{37}$

$$
\begin{aligned}
& C(\omega)=C^{\prime}(\omega)-j C^{\prime \prime}(\omega) \\
& C^{\prime}(\omega)=\frac{-Z^{\prime \prime}(\omega)}{\omega|Z(\omega)|^{2}} \\
& C^{\prime \prime}(\omega)=\frac{Z^{\prime}(\omega)}{\omega|Z(\omega)|^{2}}
\end{aligned}
$$

where $Z$ is the complex impedance written as $Z(\omega)=Z^{\prime}(\omega)+j Z^{\prime \prime}(\omega), \quad \omega=2 \pi f, \quad Z^{\prime} \quad$ and $\quad Z^{\prime \prime}$ represents the real and imaginary parts of the Nyquist plot respectively.

Using the above formulae, the capacitance of the GF/PVA/F cell is $0.62 \mathrm{~F} \mathrm{~cm}^{-2}$ as shown in Figure 6 (b). It is observed that the addition of phenol to the same material increases the specific capacitance to $1.92 \mathrm{~F} \mathrm{~cm}^{-2}$, this is attributed to the more uniformly distributed porous cavities in the electrode material, as observed from the SEM micrographs in Figure 2 (c) and (d). As a result, it promotes electrolyte ion intercalation and may slow down the diffusion of electrolyte throughout the inner pore channels, leading to an increase in capacitance due to the fast transport of ions at the electrodelelectrolyte interface.

As mentioned earlier, the short Warburg region enhances ion diffusion. This can also be explained by the diffusion process that takes place as seen in Figure 6 (c) which shows the plot of $C^{\prime \prime}$ against the frequency. The frequency $f_{0}$, which corresponds to the maximum $C^{\prime \prime}$, is defined as the transition frequency between a pure capacitive and a pure resistive behavior of the material. For GF/PVA/F and GF/PVA/FP relaxation times of $\tau=1 / f_{0}=4 \mathrm{~s}$ and $\sim 14 \mathrm{~s}$ were obtained respectively. These values show that the cells can be fully discharged within a very short time with an efficiency of more than $50 \%{ }^{6}$ It is observed that when phenol is added, it takes a longer time for the cell to become fully discharged. This drawback could be attributed to the size of the porous electrode which store charge in a distributed fashion and may not respond when an AC-frequency is applied and hence behaves more like a resistor than a capacitor. ${ }^{43}$

This can be further explained by mean of the Bode plot as shown in Figure 6 (d) which presents the phase angle as a function of frequency for both GF/PVA/F and GF/PVA/FP. In the lower frequency range, the phase angle for the electrodes is about $-84^{\circ}$ for GF/PVA/F and $-81^{\circ}$ for GF/PVA/FP. These angles are very close to $-90^{\circ}$ which defines ideal capacitors. This shows an almost pure resistive-capacitive behavior. ${ }^{44}$ The deviation of the phase angle could be attributed to the resistive part in the high frequency range (R vs. $1 / j 2 \pi C$ ), ${ }^{20}$ and also to the fact that the concentration polarization of the electrode material is cancelled by reacting species diffusing from an accumulation side to a depletion side in the lower frequency range, which drops the phase angle significantly from $90^{\circ}$, leaving the cell to act like a resistor. ${ }^{45}$

\section{Conclusions}

Highly porous three dimensional activated materials based on graphene foam and PVA polymer were investigated as electrode materials for electrochemical capacitors in aqueous electrolyte. This unique architecture of highly porous materials provides a facile, scalable, and low-cost opportunity for fabricating energy storage devices. Of the two materials characterized, GF/PVA/FP is the most promising due to its superior areal capacitance value and excellent charge propagation. The electrochemical impedance characteristic for this material showed a phase angle of $81^{\circ}$ and a relaxation time constant of $14 \mathrm{~s}$ which usually occurs at a phase angle of $45^{\circ}$ and corresponds to a transition state for supercapacitors between a pure resistive behavior at frequencies higher than $1 / \tau_{0}$ and a pure capacitive behavior for frequencies lower than $1 / \tau_{0} \cdot{ }^{37}$ These results are both interesting and encouraging, and demonstrate exciting potential for a low-cost porous carbon which could be ideal for use in high-performance supercapacitors. The fabrication of the electrode materials has not yet been fully optimized. Further 
increases in capacitance and frequency response are likely through optimization of the design of the electrode, rational selection of the electrolyte with an ionic or organic liquid, which would increase the cell's operating potential window and the use of an asymmetric cell.

\section{Acknowledgements}

This work is based upon research supported by the South African Research Chairs Initiative of the South African Department of Science and Technology (SARCHi-DST) and the National Research Foundation (NRF). Any opinion, findings and conclusions or recommendations expressed in this work are those of authors and therefore the NRF and DST do not accept any liability with regard thereto. A. Bello, acknowledges University of Pretoria and NRF financial support for his Postdoc fellowship, while D. Y. Momodu and F. Barzegar acknowledge financial support from University of Pretoria and the NRF for PhD bursaries.

\section{References}

1. J. R. Miller and P. Simon, Sci. Mag., 2008, 321, 651-652.

2. P. Simon and Y. Gogotsi, Acc. Chem. Res., 2012, 46, 10941103.

3. O. Barbieri, M. Hahn, A. Herzog, and R. Kötz, Carbon, 2005, 43, 1303-1310.

4. J. Chmiola, C. Largeot, P.-L. Taberna, P. Simon, and Y. Gogotsi, Science., 2010, 328, 480-483.

5. J. Chmiola, C. Largeot, P.-L. Taberna, P. Simon, and Y. Gogotsi, Angew. Chem. Int. Ed. Engl., 2008, 47, 3392-5.

6. D. Pech, M. Brunet, H. Durou, P. Huang, V. Mochalin, Y. Gogotsi, P.-L. Taberna, and P. Simon, Nat. Nanotechnol., 2010, 5, 651-654.

7. A. Izadi-Najafabadi, S. Yasuda, K. Kobashi, T. Yamada, D. N. Futaba, H. Hatori, M. Yumura, S. Iijima, and K. Hata, Adv. Mater., 2010, 22, E235-E241.

8. D. N. Futaba, K. Hata, T. Yamada, T. Hiraoka, Y. Hayamizu, Y. Kakudate, O. Tanaike, H. Hatori, M. Yumura, and S. Iijima, Nat. Mater., 2006, 5, 987-994.

9. Y. Wang, Z. Shi, Y. Huang, Y. Ma, C. Wang, M. Chen, and Y. Chen, J. Phys. Chem. C, 2009, 113, 13103-13107.

10. Y. Zhu, S. Murali, M. D. Stoller, K. J. Ganesh, W. Cai, P. J. 28 Ferreira, A. Pirkle, R. M. Wallace, K. A. Cychosz, M. Thommes, D. Su, E. A. Stach, and R. S. Ruoff, Science, 2011, 332, 1537-41.

11. K. Sheng, Y. Sun, C. Li, W. Yuan, and G. Shi, Sci. Rep., 2012, 2, 247.
12. Y. Fang, B. Luo, Y. Jia, X. Li, B. Wang, Q. Song, F. Kang, and L. Zhi, Adv. Mater., 2012, 24, 6348-6355.

13. Y. Wang, G. Zhao, S. Chai, H. Zhao, and Y. Wang, ACS Appl. Mater. Interfaces, 2013, 5, 842-852.

14. J. Feng, C. Zhang, J. Feng, Y. Jiang, and N. Zhao, ACS Appl. Mater. Interfaces, 2011, 3, 4796-4803.

15. L. Wei, M. Sevilla, A. B. Fuertes, R. Mokaya, and G. Yushin, Adv. Energy Mater., 2011, 1, 356-361.

16. H. Nishihara and T. Kyotani, Adv. Mater., 2012, 24, 44734498 .

17. L. Zhang, F. Zhang, X. Yang, G. Long, Y. Wu, T. Zhang, K. Leng, Y. Huang, Y. Ma, A. Yu, and C. Yongsheng, Sci. Rep., 2013, 3.

18. H. Yamada, I. Moriguchi, and T. Kudo, J. Power Sources, 2008, 175, 651-656.

19. C. Liu, Z. Yu, D. Neff, A. Zhamu, and B. Z. Jang, Nano Lett., 2010, 10, 4863-4868.

20. G. Ren, X. Pan, S. Bayne, and Z. Fan, Carbon, 2014, 71, 94-101.

21. B. Dyatkin, V. Presser, M. Heon, M. R. Lukatskaya, M. Beidaghi, and Y. Gogotsi, ChemSusChem, 2013, 6, 22692280 .

22. N. A. Choudhury, S. Sampath, and A. K. Shukla, Energy Environ. Sci., 2009, 2, 55-67.

23. T. Cai, M. Zhou, D. Ren, G. Han, and S. Guan, J. Power Sources, 2013, 231, 197-202.

24. K. Zhang, B. T. Ang, L. L. Zhang, X. S. Zhao, and J. Wu, J. Mater. Chem., 2011, 21, 2663-2670.

25. A. Bello, O. O. Fashedemi, J. N. Lekitima, M. Fabiane, D. Dodoo-Arhin, K. I. Ozoemena, Y. Gogotsi, A. T. Charlie Johnson, and N. Manyala, AIP Adv., 2013, 3, 82118.

26. G. A. Snook, P. Kao, and A. S. Best, J. Power Sources, 2011, 196, 1-12.

27. X. Zhang, W. Yang, and Y. Ma, Electrochem. Solid-State Lett., 2009, 12, A95-A98.

Y. Gogotsi and P. Simon, Science, 2011, 334, 917-8.

29. M. D. Stoller and R. S. Ruoff, Energy Environ. Sci., 2010, 3, 1294-1301.

30. K. Fic, G. Lota, M. Meller, and E. Frackowiak, Energy Environ. Sci., 2012, 5, 5842-5850. 
31. M. A. Pimenta, G. Dresselhaus, M. S. Dresselhaus, L. G. Cancado, A. Jorio, and R. Saito, Phys. Chem. Chem. Phys., 2007, 9, 1276-1290.

32. Z. Ni, T. Yu, Z. Luo, Y. Wang, and L. Liu, ACS Nano, 2009, 3, 569-574.

33. A. C. Ferrari, J. C. Meyer, V. Scardaci, C. Casiraghi, M. Lazzeri, F. Mauri, S. Piscanec, D. Jiang, K. S. Novoselov, S. Roth, and A. K. Geim, Phys. Rev. Lett., 2006, 97, 187401.

34. S. E. Denmark and J. Fu, Chem. Rev., 2003, 103, 27632794.

35. H. Zhao, F. Wang, Y. Ning, B. Zhao, F. Yin, Y. Lai, J. Zheng, X. Hu, T. Fan, J. Tang, others, D. Zhang, and K. Hu, Sci. Rep., 2013, 3, 1511.

36. B. G. Choi, M. Yang, W. H. Hong, J. W. Choi, and Y. S. Huh, ACS Nano, 2012, 6, 4020-4028.

37. P. L. Taberna, P. Simon, and J.-F. Fauvarque, $J$. Electrochem. Soc., 2003, 150, A292-A300.

38. F. Lufrano, P. Staiti, T. Avanzate, and E. Nicola, Int J Electrochem Sci, 2010, 5, 903-916.

39. W. Wang, S. Guo, M. Ozkan, and C. S. Ozkan, J. Mater. Res., 2013, 28, 912-917.

40. J. R. McDonough, J. W. Choi, Y. Yang, F. La Mantia, Y. Zhang, and Y. Cui, Appl. Phys. Lett., 2009, 95, 243109.

41. B. G. Choi, J. Hong, W. H. Hong, P. T. Hammond, and H. Park, ACS Nano, 2011, 5, 7205-7213.

42. E. Lust, A. Jänes, and M. Arulepp, J. Electroanal. Chem., 2004, 562, 33-42.

43. J. R. Miller, R. A. Outlaw, and B. C. Holloway, Science, 2010, 329, 1637-1639.

44. E. Barsoukov and J. R. Macdonald, Impedance spectroscopy: theory, experiment, and applications, John Wiley \& Sons, 2005.

45. H. Ji, Y. Mei, and O. G. Schmidt, Chem. Commun., 2010, 46, 3881-3883.

\section{Notes}

Department of Physics, Institute of Applied Materials, SARChI Chair in Carbon Technology and Materials, University of Pretoria, Pretoria 0028, South Africa.

*Corresponding author. E-mail address: bellohakeem@gmail.com and ncholu.manyala@up.ac.za

Tel.: +27 012420 3549; Fax: +27 0124202516 (N. Manyala) 